Rev. Biol. Neotrop. 5(1): 1-16, 2008

\title{
ntóceros e Hepáticas do Parque Estadual da Serra dos Pireneus e arredores, Município de Pirenópolis, Goiás, Brasil
}

\author{
Mariella Abrahão Rodrigues de Sousa \\ Vera lúcia Gomes Klein \\ Maria Helena Rezende
}

Departamento de Biologia Geral, Instituto de Ciências Biológicas, Universidade Federal de Goiás, Campus II, Goiânia, Goiás, Brasil, 74001-970; e-mail: vlgomes.ufg@gmail.com

Olga Yano

Instituto de Botânica, Caixa Postal 3005, São Paulo, São Paulo, Brasil, 01061-970

\begin{abstract}
Resumo: O Parque Estadual da Serra dos Pireneus localiza-se nos municípios de Pirenópolis, Cocalzinho e Corumbá de Goiás, no estado de Goiás. Este trabalho teve como objetivos principais a elaboração de uma lista de antóceros e hepáticas ocorrentes no parque e adjacências e a verificação de sua distribuição geográfica no Brasil. A região compreende vegetação de cerrado sensu stricto, campo limpo, campo sujo, floresta úmida semidecídua, floresta de galeria e formações rupestres. Foram coletadas nessa serra uma espécie de Anthocerotophyta e 57 espécies de Marchantiophyta distribuídas em 29 gêneros e 14 famílias, sendo as mais representativas: Lejeuneaceae (19 sp.), Jubulaceae (8 sp.), Lepidoziaceae (7 sp.) e Plagiochilaceae ( $5 \mathrm{sp}$.). Entre as plantas coletadas durante esta pesquisa, 38 espécies são de ocorrência nova para o estado de Goiás. Portanto, a Serra dos Pireneus é uma região que apresenta grande diversidade de briófitas e o conhecimento acerca delas contribuirá para a conservação da área e o estudo da brioflora do estado.
\end{abstract}

Palavras-chave: Antóceros, Brasil, distribuição geográfica, Goiás, hepáticas.

Abstract: Parque Estadual da Serra dos Pireneus is located in Pirenópolis, Cocalzinho, and Corumbá de Goiás, in the state of Goiás. This work aimed to prepare a list of the liverworts and hornworts that occur in that park and verify their geographical distribution within Brazil. In this region, there are several vegetation types: cerrado sensu stricto, campo limpo (shrubfree grassland), campo sujo (grassland with scattered shrubs), semideciduous forest, gallery forest, and rocky outcrop formations. During this research, it was possible to find in this mountain range one species of Anthocerotophyta and 57 species of Marchantiophyta distributed in 29 genera and 14 families, among which the most representative ones are: Lejeuneaceae (19 sp.), Jubulaceae (8 sp.), Lepidoziaceae (7 sp.), and Plagiochilaceae (5 sp.). Among the plants collected, 38 species are new records for the state of Goiás. Therefore, Serra dos Pireneus is a region that presents high diversity of bryophytes and the knowledge about them is definitely going to contribute to the conservation and study of the bryoflora of the state.

KeY woRDs: Liverworts, Brazil, geographical distribution, Goiás, hornworts.

\section{INTRODUÇÃO}

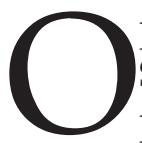
primeiro relato sobre briófitas na Serra dos Pireneus foi publicado por Brotherus (1895), no qual o autor descreveu Octoblepharum rhaphidostegium Müll. Hal, material coletado por Ule em 1893, além de relacionar 55 espécies de musgos para o estado.

Yano \& Costa (2000) relataram 10 espécies de briófitas para os estados de Goiás e Tocantins; Gradstein et al. (2005) listaram 64 espécies de hepáticas para Goiás, um estado para o qual ainda há poucos estudos acerca de briófitas, bem como coletas de exemplares desta divisão de plantas.

Este trabalho teve como objetivos a elaboração de uma lista de espécies de antóceros e hepáticas ocorrentes na Serra dos Pireneus e a verificação de sua distribuição no Brasil. 


\section{Material e métodos}

O Parque Estadual da Serra dos Pireneus localiza-se nos municípios de Pirenópolis, Cocalzinho e Corumbá de Goiás, aproximadamente entre $15^{\circ} 50^{\prime} \mathrm{S}$ e $48^{\circ} 47^{\prime} \mathrm{W}$, com altitude máxima de $1.385 \mathrm{~m}$ no Pico dos Pireneus. A vegetação da área compreende regiões de cerrado sensu stricto, campo limpo, campo sujo, floresta úmida semidecídua, floresta de galeria e formações rupestres.

Todas as coletas foram realizadas no município de Pirenópolis, dentro do Parque Estadual da Serra dos Pireneus ou em seus imediatos arredores, em duas viagens, realizadas em 2 de outubro de 2005 e 18-19 de março de 2006.

A identificação das amostras foi baseada nos trabalhos de Bastos (2004), Castle (1964-1965), Costa (1999), Costa \& Moura (1996), Fulford (1963, 1966, 1968, 1976), Gradstein (1994), Gradstein \& Costa (2003), Heinrichs \& Gradstein (2000), Heinrichs et al. (1998), Hell (1969), Kuwahara (1986), Lemos-Michel (2001), Lindenberg (1839-1844), Oliveira e Silva \& Yano (2000), Reiner-Drehwald (2000), Schuster (1969, 1980), Schuster \& Blomquist (1955), Stotler (1970), Tixier (1980, 1985, 1991), Vá a (1975), Vanden Berghen (1976) e Yano \& Mello (1999). A distribuição geográfica brasileira das espécies citadas baseou-se nos trabalhos de Yano (1984, 1989, 1995, 2006) e Gradstein et al. (2005). O sistema de classificação de antóceros foi baseado em Stotler \& Crandall-Stotler (2005) e o de hepáticas, em Crandall-Stotler \& Stotler (2000).

\section{Resultados}

No Parque Estadual da Serra dos Pireneus e arredores foram encontradas 58 espécies de hepáticas e antóceros, sendo uma de Anthocerotophyta e 57 de Marchantiophyta, distribuídas em 29 gêneros e 14 famílias.

A seguir, todas essas espécies são listadas em ordem alfabética por família, gênero e espécie nas Divisões Anthocerotophyta e Marchantiophyta, e comentadas com observações ecológicas, ilustrações disponíveis e material examinado.

\section{ANTHOCEROTOPHYTA}

\section{AnthocerotaceAe}

*Phaeoceros laevis (L.) Prosk., Bull. Torrey Bot. Club 78(4): 346, 1951. Anthoceros laevis L.

Ilustrações: Gradstein \& Costa (2003) e Hässel de Menéndez (1962).

Material examinado: Município de Pirenópolis, Parque Estadual da Serra dos Pireneus, sobre o solo, em barranco próximo a um rego d'água que corta a estrada rumo à Cachoeira de São Lázaro, 19.III.2006, M. A. R. Sousa \& V. L. Gomes-Klein 124 (UFG 30202).

No Brasil, ocorre no Distrito Federal e nos estados de Amazonas, Bahia, Espírito Santo, Mato Grosso do Sul, Minas Gerais, Pernambuco, Rio Grande do Sul, Rio de Janeiro, Santa Catarina e São Paulo.

*Primeira referência para Goiás.

\section{MARCHANTIOPHYTA}

\section{Acrobolbaceae}

*Lethocolea glossophylla (Spruce) Grolle, Bot. Mag. Tokyo 78: 83, 1965. Symphyomitra glossophylla Spruce.

Ilustração: Gradstein \& Costa (2003).

Material examinado: Município de Pirenópolis, Parque Estadual da Serra dos Pireneus, Morro do Cabeludo, no solo, perto da base do tronco, 18.III.2006, O. Yano $\mathcal{E} M$. A. R. Sousa 28670 (SP 380005).

No Brasil, ocorre nos estados de Espírito Santo e Minas Gerais.

*Primeira referência para Goiás.

\section{Aneuraceae}

*Riccardia cataractarum (Spruce) Hell, Bol. Univ. São Paulo. Bot. 25: 97, 1969. Aneura cataractarum Spruce.

Ilustração: Hell (1969).

Material examinado: Município de Pirenópolis, Parque Estadual da Serra dos Pireneus, rupícola, 2.X.2005, M. A. R. Sousa $\mathcal{E} A$. Moris 13 (UFG 30091); idem, na estrada lateral de Pirenópolis a Cocalzinho, ca. $8 \mathrm{~km}$ a $9 \mathrm{~km}$ do entroncamento, no barranco úmido perto da estrada, 19.III.2006, O. Yano \& M. A. R. Sousa 28802 (SP 382905). 
No Brasil, ocorre no Distrito Federal e nos estados de Espírito Santo, Mato Grosso, Mato Grosso do Sul, Minas Gerais, Rio de Janeiro, Santa Catarina e São Paulo.

*Primeira referência para Goiás.

\section{BALANTIOPSiDACEAE}

*Neesioscyphus homophyllus (Nees) Grolle, Österr. Bot. Zeitschr. 111(2-3): 188, 1964. Jungermannia homophyllus Nees in Martius.

Ilustração: Gradstein \& Costa (2003).

Material examinado: Município de Pirenópolis, Parque Estadual da Serra dos Pireneus, sobre o solo, em barranco próximo a um rego d'água que corta a estrada rumo à Cachoeira de São Lázaro, 19.III.2006, M. A. R. Sousa \& V. L. Gomes-Klein 126 (UFG 30204).

No Brasil, ocorre nos estados de Minas Gerais, Rio de Janeiro e São Paulo.

*Primeira referência para Goiás.

\section{Cephaloziaceae}

${ }^{*}$ Cephalozia crassifolia (Lindenb. \& Gott.) Fulf., Mem. New York Bot. Gard. 11: 312, 1968. Jungermannia crassifolia Lindenb. \& Gott.

Ilustração: Fulford (1968).

Material examinado: Município de Pirenópolis, Parque Estadual da Serra dos Pireneus, na base do Morro do Cabeludo, sobre pedra úmida, 18.III.2006, O. Yano E M. A. R. Sousa 28701 p.p. (SP 380036); idem, em solo úmido, 18.III.2006, O. Yano \& M. A. R. Sousa 28729 p.p. (SP 382833).

Cresce associada com Odontoschisma brasiliense Hampe e Telaranea nematodes (Gott. ex Aust.) Howe.

No Brasil, ocorre nos estados de Bahia, Espírito Santo, Rio de Janeiro, Santa Catarina e São Paulo.

*Primeira referência para Goiás.

*Odontoschisma brasiliense Steph., Bull. Herb. Boissier. ser 2, 8: 585, 1988.

Ilustração: Fulford (1968).

Material examinado: Município de Pirenópolis, Parque Estadual da Serra dos Pireneus, na estrada para o Morro do Cabeludo, no barranco do riacho, 18.III.2006, O. Yano $\mathcal{E}$ M. A. R. Sousa 28622 (SP 379958); idem, na base do Morro do Cabeludo, sobre pedra úmida,
18.III.2006, O. Yano \& M. A. R. Sousa 28701 p.p. (SP 380036), 28705 p.p. (SP 380040); idem, sobre barranco, no leito do riacho, na estrada para o Sítio Vereda de Cristal, 18.III.2006, M. A. R. Sousa \& V. L. Gomes-Klein 46 (UFG 30124).

Cresce associada com Cephalozia crassifolia (Lindenb. \& Gott.) Fulf. e Kurzia brasiliensis (Sw.) Grolle.

No Brasil, ocorre nos estados de Amazonas, Minas Gerais e Rio de Janeiro.

*Primeira referência para Goiás.

Odontoschisma longiflorum (Tayl.) Steph., Spec. Hepat. 3: 370, 1908. Sphagnoecetes longiflora Tayl.

Ilustração: Fulford (1968).

Material examinado: Município de Pirenópolis, Parque Estadual da Serra dos Pireneus, na base do Morro do Cabeludo, sobre pedra úmida, 18.III.2006, O. Yano $\mathcal{E}$ M. A. R. Sousa 28738 p.p.(SP 382842).

Cresce associada com Syrrhopodon prolifer Schwägr.

No Brasil, ocorre nos estados de Goiás (Chapada dos Veadeiros), Mato Grosso, Minas Gerais, Paraná, Rio de Janeiro, Santa Catarina e São Paulo.

\section{FosSOMBRONIACEAE}

*Fossombronia porphyrorhiza (Nees) Prosk., Bryologist 58: 197, 1955. Jugermannia porphyrorhiza Nees in Martius.

Ilustração: Gradstein \& Costa (2003).

Material examinado: Município de Pirenópolis, sítio Vereda de Cristal, no barranco úmido perto do Chalé Beira Rio, 18.III.2006, O. Yano E M. A. R. Sousa 28599 (SP 379935); idem, na estrada 2 para o Morro do Cabeludo, no barranco úmido, 18.III.2006, O. Yano \& M. A. R. Sousa 28618 (SP 379954); idem, na estrada lateral de Pirenópolis a Cocalzinho, ca. $8 \mathrm{~km}$ a $9 \mathrm{~km}$ do entroncamento, no solo úmido da mata, 19.III.2006, O. Yano \& M. A. R. Sousa 28793 (SP 382897); idem, Parque Estadual da Serra dos Pireneus, rupícola, na mata da base do Morro do Cabeludo, 18.III.2006, M. A. R. Sousa \& V. L. Gomes-Klein 62 (UFG 30140), 92 (UFG 30170); idem, sobre o solo, em barranco próximo ao rego d'água que corta a estrada rumo à Fazenda Portal do Sol e Lázaro, 19.III.2006, M. A. R. Sousa \& V. L. Gomes-Klein 112 (UFG 30190). 
No Brasil, ocorre no Distrito Federal e nos estados de Bahia, Ceará, Espírito Santo, Mato Grosso, Mato Grosso do Sul, Minas Gerais, Pernambuco, Rio de Janeiro e São Paulo.

*Primeira referência para Goiás.

Frullaniaceae/Jubulaceae

${ }^{*}$ Frullania arecae (Spreng.) Gott., Mexik. Leverm.: 236, 1863. Jungermannia arecae Spreng.

Ilustrações: Lemos-Michel (2001) e Vanden Berghen (1976).

Material examinado: Município de Pirenópolis, Córrego da Barriguda, 1.040 m alt., sobre tronco de arbusto, 18.III.2006, O. Yano $\mathcal{E}$ M. A. R. Sousa 28603 (SP 379939).

No Brasil, ocorre no Distrito Federal e nos estados de Acre, Bahia, Espírito Santo, Mato Grosso, Minas Gerais, Paraná, Rio Grande do Sul, Rio de Janeiro, Roraima, São Paulo e Sergipe.

*Primeira referência para Goiás.

${ }^{*}$ Frullania beyrichiana (Lehm. \& Lindenb.) Lindenb. in Gottsche et al., Syn. Hepat.: 460, 1845. Jungermannia beyrichiana Lehm. \& Lindenb.

Ilustrações: Lemos-Michel (2001) e Stotler (1970).

Material examinado: Município de Pirenópolis, Parque Estadual da Serra dos Pireneus, na base do Morro do Cabeludo, sobre pedra úmida, 18.III.2006, O. Yano \& M. A. R. Sousa 28699 (SP 380034).

No Brasil, ocorre nos estados de Acre, Bahia, Espírito Santo, Mato Grosso, Minas Gerais, Pará, Pernambuco, Rio Grande do Sul, Rio de Janeiro e São Paulo.

*Primeira referência para Goiás.

Frullania brasiliensis Raddi, Soc. Ital. Atti. Sci. Mod. 19: 36, 1822.

Ilustrações: Lemos-Michel (2001) e Stotler (1970).

Material examinado: Município de Pirenópolis, Parque Estadual da Serra dos Pireneus, na base do Morro do Cabeludo, sobre pedra úmida, 18.III.2006, O. Yano $\mathcal{E}$ M. A. R. Sousa 28688 (SP 380023).

No Brasil, ocorre no Distrito Federal e nos estados de Bahia, Ceará, Espírito Santo,
Goiás (Alto Paraíso, Chapada dos Veadeiros), Minas Gerais, Pernambuco, Rio Grande do Sul, Rio de Janeiro, Santa Catarina e São Paulo.

*Frullania dusenii Steph., Archos Mus. Nat. Rio de Janeiro 13: 115, 1903.

Ilustração: Gradstein \& Costa (2003).

Material examinado: Município de Pirenópolis, na estrada para o Morro do Cabeludo, sobre tronco de arbusto, 18.III.2006, O. Yano $\mathcal{E}$ M. A. R. Sousa 28620 (SP 379956); idem, Córrego da Barriguda, sobre tronco de arbusto, 18.III.2006, O. Yano \& M. A. R. Sousa 28640 (SP 379976); idem, sobre tronco vivo, em barranco da estrada rumo à Fazenda Portal do Sol e Lázaro, 19.III.2006, M. A. R. Sousa 107 (UFG 30185), 108 (UFG 30186), 114 (UFG 30192).

No Brasil, ocorre nos estados de Espírito Santo, Minas Gerais, Pernambuco, Rio Grande do Sul, Rio de Janeiro, Roraima, Santa Catarina, São Paulo e Sergipe.

*Primeira referência para Goiás.

Frullania ericoides (Nees) Mont., Ann. Sci. Nat. Bot. ser 2, 12: 51, 1839. Jungermannia ericoides Nees in Martius.

Ilustrações: Gradstein \& Costa (2003), Lemos-Michel (2001), Vanden Berghen (1976) e Yano \& Mello (1999).

Material examinado: Município de Pirenópolis, sítio Vereda de Cristal, perto da casa, sobre pedra perto do córrego no Chalé Beira Rio, 18.III.2006, O. Yano E M. A. R. Sousa 28597 p.p. (SP 379933); idem, Córrego da Barriguda, $1.040 \mathrm{~m}$ alt., na base do tronco de arbusto, 18.III.2005, O. Yano E M. A. R. Sousa 28613 (SP 379949); idem, Parque Estadual da Serra dos Pireneus, Morro do Cabeludo, sobre pedra no morro, 18.III.2006, O. Yano \& M. A. R. Sousa 28672 (SP 380007).

Cresce associada com Acrolejeunea torulosa (Lehm. \& Lindenb.) Schiffn.

No Brasil, ocorre no Distrito Federal, na Ilha Fernando de Noronha e nos estados de Acre, Amazonas, Bahia, Ceará, Espírito Santo, Goiás (Mineiros, Chapada dos Veadeiros), Mato Grosso, Mato Grosso do Sul, Minas Gerais, Paraíba, Paraná, Pernambuco, Rio Grande do Sul, Rio de Janeiro, Santa Catarina, São Paulo e Sergipe. 
*Frullania glomerata (Lehm. \& Lindenb.) Gott., Ann. Sci. Nat. Bot. ser. 2, 9: 46, 1838.

Basiônimo: Jungermannia glomerata Lehm. \& Lindenb.

Ilustração: Yano \& Mello (1999).

Material examinado: Município de Pirenópolis, na estrada para o Morro do Cabeludo, sobre tronco de arbusto, 18.III.2006, O. Yano $\mathcal{E}$ M. A. R. Sousa 28631 p.p. (SP 379967); idem, na estrada lateral de Pirenópolis a Cocalzinho, ca. $8 \mathrm{~km}$ a $9 \mathrm{~km}$ do entroncamento, sobre tronco de árvore na encosta, 19.III.2006, O. Yano E M. A. R. Sousa 28763 (SP 382867), 28768 (SP 382872); idem, sobre pedra úmida na mata, 19.III.2006, O. Yano E M. A. R. Sousa 28776 (SP 382880), 28790 (SP 382894); idem, sobre tronco de arbusto na mata, 19.III.2006, O. Yano E M. A. R. Sousa 28794 p.p. (SP 382898); idem, sobre pedra úmida na mata perto da estrada, 19.III.2006, O. Yano E M. A. R. Sousa 28810 (SP 382913).

Cresce associada com Acrolejeunea torulosa (Lehm. \& Lindenb.) Schiffn. e Cheilolejeunea rigidula (Nees \& Mont.) Schust.

No Brasil, ocorre no Distrito Federal e nos estados de Bahia, Ceará, Mato Grosso, Mato Grosso do Sul, Minas Gerais, Paraná, Rio Grande do Sul, Rio de Janeiro e São Paulo.

*Primeira referência para Goiás.

*Frullania neesii Lindenb. in Gottsche et al., Syn. Hepat.: 450, 1845.

Ilustração: Lemos-Michel (2001).

Material examinado: Município de Pirenópolis, na estrada para o Morro do Cabeludo, sobre tronco de arbusto, 18.III.2006, O. Yano E M. A. R. Sousa 28629 (SP 379965), 28634 (SP 379970); idem, córrego da Barriguda, rupícola, 2.X.2005, M. A. R. Sousa \& A. Moris 23 (UFG 30104); idem, sobre raízes perto do córrego, 18.III.2006, O. Yano E M. A. R. Sousa 28639 (SP 379975); idem, sobre tronco de arbusto, 18.III.2006, O. Yano \& M. A. R. Sousa 28642 (SP 379978); idem, Parque Estadual da Serra dos Pireneus, Morro do Cabeludo, na base do tronco de arbusto, 18.III.2006, O. Yano $\mathcal{E}$ M. A. R. Sousa 28645 (SP 379981); idem, nos galhos podres no morro, 18.III.2006, O. Yano $\mathcal{E}$ M. A. R. Sousa 28655 (SP 379991); idem, sobre canela-de-ema na pedra, 18.III.2006, O. Yano
$\mathcal{E}$ M. A. R. Sousa 28662 (SP 379997); idem, sobre pedra úmida, 18.III.2006, O. Yano $\mathcal{E}$ M. A. R. Sousa 28674 (SP 380009), 28678 (SP 380013); idem, na base do Morro do Cabeludo, sobre tronco de arbusto, 18.III.2006, O. Yano $\mathcal{E}$ M. A. R. Sousa 28695 (SP 380030); idem, na estrada lateral de Pirenópolis a Cocalzinho, ca. $8 \mathrm{~km}$ a $9 \mathrm{~km}$ do entroncamento, sobre tronco de $\mathrm{Me}$ lastomataceae na encosta, 19.III.2006, O. Yano E M. A. R. Sousa 28775 (SP 382879); idem, na base do tronco de arbusto na mata, 19.III.2006, O. Yano E M. A. R. Sousa 28788 (SP 382892); idem, sobre pedra úmida na mata, 19.III.2006, O. Yano E M. A. R. Sousa 28807 (SP 382910); idem, rupícola, na base da mata do Morro do Cabeludo, 18.III.2006, M. A. R. Sousa \& V. L. Gomes-Klein 59 (UFG 30137); idem, na base de tronco vivo, próximo a riacho, na estrada para o Sítio Vereda de Cristal, 18.III.2006, M. A. R. Sousa \& V. L. Gomes-Klein 39 (UFG 30117).

Cresce associada com Sematophyllum subpinnatum (Brid.) Britt.

No Brasil, ocorre no Distrito Federal e nos estados de Acre, Amazonas, Bahia, Ceará, Espírito Santo, Mato Grosso, Minas Gerais, Paraíba, Paraná, Pernambuco, Rio Grande do Sul, Rio de Janeiro, Roraima, São Paulo e Sergipe.

*Primeira referência para Goiás.

Frullania riojaneirensis (Raddi) Ångstr., Öfvers K. VetenskAkad. Förh. 33(7): 88, 1876. Frullanoides riojaneiresnsis Raddi.

Ilustração: Gradstein \& Costa (2003).

Material examinado: Município de Pirenópolis, Parque Estadual da Serra dos Pireneus, Morro do Cabeludo, sobre pedras no morro, 18.III.2006, O. Yano \& M. A. R. Sousa 28676 (SP 380011); idem, na estrada lateral de Pirenópolis a Cocalzinho, ca. $8 \mathrm{~km}$ a $9 \mathrm{~km}$ do entroncamento, sobre pedra úmida na mata, 19.III.2006, O. Yano E M. A. R. Sousa 28789 (SP 382893).

No Brasil, ocorre no Distrito Federal e nos estados de Bahia, Ceará, Espírito Santo, Goiás (Cristalina; Serra Dourada; Alto Paraíso de Goiás; Goiânia, Campus II), Mato Grosso, Mato Grosso do Sul, Minas Gerais, Pará, Paraíba, Paraná, Pernambuco, Rio Grande do Sul, Rio de Janeiro, Santa Catarina, São Paulo e Sergipe. 
Geocalycaceae

Lophocolea mandonii Steph., Bull. Herb. Boissier. ser. 2, 7: 61, 1907.

Ilustração: Fulford (1976).

Material examinado: Município de Pirenópolis, Parque Estadual da Serra dos Pireneus, sobre o solo, em barranco próximo ao rego d'água que corta a estrada rumo à Cachoeira de São Lázaro, 19.III.2006, M. A. R. Sousa \& V. L. Gomes-Klein 122 (UFG 30200).

No Brasil, ocorre nos estados de Bahia, Espírito Santo, Goiás (Itaberaí, município de Formoso), Minas Gerais e Rio de Janeiro.

*Lophocolea martiana Nees in Gottsche et al., Syn. Hepat.: 152, 1845.

Ilustrações: Fulford (1976) e Lemos-Michel (2001).

Material examinado: Município de Pirenópolis, Parque Estadual da Serra dos Pireneus, quase dentro do Córrego da Barriguda, rupícola, 2.X.2005, M. A. R. Sousa $\mathcal{E}$ A. Moris 14 (UFG 30092); idem, na base do Morro do Cabeludo, no solo da mata, 18.III.2006, O. Yano E M. A. R. Sousa 28714 (SP 382818), 28744 (SP 382848).

No Brasil, ocorre nos estados de Amazonas, Amapá, Bahia, Ceará, Espírito Santo, Mato Grosso, Minas Gerais, Pará, Paraná, Pernambuco, Rio Grande do Sul, Rio de Janeiro, Santa Catarina, São Paulo e Sergipe.

*Primeira referência para Goiás.

*Lophocolea platensis C. Massal., Atti Acad. Sci Med. Nat-Ferrara 80(3-4): 21, 1906.

Ilustração: Gradstein \& Costa (2003).

Material examinado: Município de Pirenópolis, Parque Estadual da Serra dos Pireneus, na base do Morro do Cabeludo, sobre pedra úmida, 18.III.2006, O. Yano E M. A. R. Sousa 28736 (SP 382840); idem, sobre rocha dentro do riacho, 18.III.2006, M. A. R. Sousa \& V. L. Gomes-Klein 57 p.p. (UFG 30135); idem, rupícola, dentro do rio que corta a base do Morro do Cabeludo, 18.III.2006, M. A. R. Sousa \& V. L. Gomes-Klein 76 p.p. (UFG 30154), 77 p.p. (UFG 30155); idem, sobre o solo, em barranco da estrada rumo à Fazenda Portal do Sol e Lázaro, 19.III.2006, M. A. R. Sousa \& V. L. Gomes-Klein 109 (UFG 30187), 120 (UFG 30198).
Cresce associada com Symphyogyna aspera Steph. e Taxilejeunea pterigonia (Lehm. \& Lindenb.) Schiffn.

No Brasil, ocorre nos estados de Bahia, Espírito Santo, Mato Grosso, Santa Catarina e São Paulo.

*Primeira referência para Goiás.

JUNGERMANNIACEAE

*Jungermannia hyalina Lyell in Hooker, Brit. Jungerm. tab: 63, 1814.

Ilustrações: Gradstein \& Costa (2003), Schuster (1969, como Solenostoma hyalinum (Lyell in Hook.) Mitt.) e Váňa (1975).

Material examinado: Município de Pirenópolis, na estrada lateral de Pirenópolis a Cocalzinho, ca. $8 \mathrm{~km}$ a $9 \mathrm{~km}$ do entroncamento, no solo úmido da mata, 19.III.2006, O. Yano E M. A. R. Sousa 28799 (SP 382902); idem, no barranco perto da estrada, 19.III.2006, O. Yano E M. A. R. Sousa 28805 p.p. (SP 382908), 28808 (SP 382911).

Cresce associada com Paracromastigum pachyrhizum (Nees) Fulf.

No Brasil, ocorre nos estados de Mato Grosso, Mato Grosso do Sul, Minas Gerais, Piauí e Rio de Janeiro.

*Primeira referência para Goiás.

\section{LeJEUNEACEAE}

*Acrolejeunea torulosa (Lehm. \& Lindenb.) Schiffn. in Engler \& Prantl, Natürl. Pflanzenfam. 1(3): 128, 1893. Jungermannia torulosa Lehm. \& Lindenb. in Lehmann.

Ilustração: Gradstein (1994).

Material examinado: Município de Pirenópolis, sítio Vereda de Cristal, perto da casa, sobre pedra perto do córrego no Chalé Beira Rio, 18.III.2006, O. Yano \& M. A. R. Sousa 28597 p.p. (SP 379933); idem, na estrada para o Morro do Cabeludo, sobre tronco de Clusiaceae, 18.III.2006, O. Yano \& M. A. R. Sousa 28631 p.p. (SP 379967); idem, na base do tronco, 18.III.2006, O. Yano E M. A. R. Sousa 28632 (SP 379968).

Cresce associada com Frullania ericoides (Nees) Mont. e F. glomerata (Lehm. \& Lindenb.) Mont.

No Brasil, ocorre nos estados de Acre, Alagoas, Amazonas, Bahia, Ceará, Espírito Santo, 
Maranhão, Mato Grosso, Mato Grosso do Sul, Minas Gerais, Pará, Paraná, Rio de Janeiro, Rondônia, Roraima e São Paulo.

*Primeira referência para Goiás.

Aphanolejeunea truncatifolia Horik., J. Sci. Hiroshima Univ. ser. 3. Div. 2, 2: 284, 1934.

Ilustrações: Gradstein \& Costa (2003) e Schuster (1980, como Aphanolejeunea diaphana (Evans) Schust.).

Material examinado: Município de Pirenópolis, Parque Estadual da Serra dos Pireneus, na base do Morro do Cabeludo, epífila de arbusto na mata, 18.III.2006, O. Yano $\mathcal{E} M$. A. R. Sousa 28760 p.p. (SP 382864), 28761 p.p. (SP 382865).

Cresce associada com Cololejeunea cardiocarpa (Mont.) Evans e C. subcardiocarpa P. Tixier.

No Brasil, ocorre no Distrito Federal e nos estados de Amazonas, Bahia, Espírito Santo, Goiás (Alto Paraíso, Chapada dos Veadeiros), Mato Grosso, Mato Grosso do Sul, Minas Gerais, Pará, Pernambuco, Rio Grande do Sul, Rio de Janeiro, Santa Catarina e São Paulo.

Cheilolejeunea clausa (Nees \& Mont.) Schust., Hepat. Anthocerotae N. Amer. 4: 863, 1980. Lejeunea clausa Nees \& Mont. in Montagne.

Ilustração: Schuster (1980).

Material examinado: Município de Pirenópolis, Parque Estadual da Serra dos Pireneus, na base do Morro do Cabeludo, sobre pedra úmida, 18.III.2006, O. Yano E M. A. R. Sousa 28685 (SP 380020); idem, sobre tronco podre, 18.III.2006, O. Yano \& M. A. R. Sousa 28692 p.p. (SP 380027); idem, sobre tronco de arbusto na mata, 18.III.2006, O. Yano $\mathcal{E}$ M. A. R. Sousa 28747 (SP 382851).

Cresce associada com Lejeunea laetevirens Nees \& Mont.

No Brasil, ocorre nos estados de Acre, Amazonas, Amapá, Bahia, Ceará, Espírito Santo, Goiás (Itaberaí; Formoso; Aporé; Goiânia, Jardim Botânico), Mato Grosso, Mato Grosso do Sul, Minas Gerais, Pará, Paraná, Pernambuco, Rio de Janeiro, Santa Catarina e São Paulo.

Cheilolejeunea rigidula (Nees \& Mont.) Schust., Castanea 36: 102, 1971. Lejeunea rigidula Mont.

Ilustração: Schuster (1980).
Material examinado: Município de Pirenópolis, Córrego da Barriguda, $1.040 \mathrm{~m}$ alt., epífita, 2.X.2005, M. A. R. Sousa \& A. Moris 22 p.p. (UFG 30098); idem, na base do tronco de canela-de-ema, 18.III.2006, O. Yano $\mathcal{E}$ M. A. R. Sousa 28614 p.p. (SP 379950); idem, na estrada para o Morro do Cabeludo, sobre tronco de arbusto, 18.III.2006, O. Yano E M. A. R. Sousa 28623 p.p. (SP 379959); idem, Parque Estadual da Serra dos Pireneus, Morro do Cabeludo, sobre tronco vivo de Clusiaceae, 18.III.2006, O. Yano E M. A. R. Sousa 28666 (SP 380001); idem, na base do Morro do Cabeludo, sobre tronco de árvore, 18.III.2006, O. Yano $\mathcal{E}$ M. A. R. Sousa 28691 p.p. (SP 380026); idem, sobre galhos finos de arbusto, 18.III.2006, O. Yano $\mathcal{E} M$. A. R. Sousa 28735 p.p. (SP 382839); idem, sobre tronco de arbusto na mata, 19.III.2006, O. Yano E M. A. R. Sousa 28794 p.p. (SP 382898), 28797 (SP 382900); idem, rupícola, na base da mata do Morro do Cabeludo, 18.III.2006, M. A. R. Sousa \& V. L. Gomes-Klein 85 p.p. (UFG 30163); idem, sobre tronco caído, na base da mata do Morro do Cabeludo, 18.III.2006, M. A. R. Sousa \& V. L. Gomes-Klein 91 (UFG 30169).

Cresce associada com Brachymenium exile (Dozy \& Molk) Bosch \& Lac., Frullania glomerata (Lehm. \& Lindenb.) Mont., Metzgeria lechleri Steph., Schiffneriolejeunea polycarpa (Nees) Gradst., Sematophyllum subpinnatum (Brid.) Britt., S. subsimplex (Hedw.) Mitt., Taxilejeunea pterigonia (Lehm. \& Lindenb.) Schiffn. e Thuidium delicatulum (Hedw.) B.S.G.

No Brasil, ocorre no Distrito Federal e nos estados de Acre, Alagoas, Amapá, Amazonas, Bahia, Ceará, Espírito Santo, Goiás (Alto Paraíso, Chapada dos Veadeiros), Mato Grosso, Mato Grosso do Sul, Minas Gerais, Pará, Paraíba, Paraná, Pernambuco, Rio de Janeiro, Roraima, Santa Catarina, São Paulo e Sergipe.

Cheilolejeunea trifaria (Reinw. et al.) Mizut., J. Hattori Bot. Lab. 27: 132, 1964. Jungermannia trifaria Reinw. et al.

Ilustrações: Gradstein \& Costa (2003) e Oliveira e Silva \& Yano (2000).

Material examinado: Município de Pirenópolis, Parque Estadual da Serra dos Pireneus, na base do Morro do Cabeludo, sobre pedra úmida, 18.III.2006, O. Yano $\mathcal{E}$ M. A. R. 
Sousa 28687 (SP 380022); idem, sobre tronco de arbusto, 18.III.2006, O. Yano E M. A. R. Sousa 28718 (SP 382822); idem, na estrada lateral de Pirenópolis a Cocalzinho, ca. $8 \mathrm{~km}$ a $9 \mathrm{~km}$ do entroncamento, na base do tronco de arbusto na encosta, 19.III.2006, O. Yano \& M. A. R. Sousa 28771 (SP 382875), 28780 (SP 382884); idem, sobre pedra úmida na mata, 19.III.2006, O. Yano E M. A. R. Sousa 28785 (SP 382889); idem, corticícola, na base da mata do Morro do Cabeludo, 18.III.2006, M. A. R. Sousa \& V. L. Gomes-Klein 84 (UFG 30162).

No Brasil, ocorre no Distrito Federal e nos estados de Acre, Amapá, Amazonas, Bahia, Ceará, Espírito Santo, Goiás (Alto Paraíso, Chapada dos Veadeiros), Mato Grosso, Mato Grosso do Sul, Minas Gerais, Pará, Paraíba, Paraná, Pernambuco, Rio de Janeiro, Roraima e São Paulo.

Cololejeunea cardiocarpa (Mont.) Evans, Mem. Torrey Bot. Club 8: 172, 1902. Lejeunea cardiocarpa Mont.

Ilustrações: Schuster (1980) e Tixier (1985).

Material examinado: Município de Pirenópolis, Parque Estadual da Serra dos Pireneus, na base do Morro do Cabeludo, epífila de arbusto na mata, 18.III.2006, O. Yano $\mathcal{E}$ M. A. R. Sousa 28721 (SP 382825), 28759 (SP 382863), 28760 p.p. (SP 382864$) ;$ idem, na estrada lateral de Pirenópolis a Cocalzinho, ca. $8 \mathrm{~km}$ a $9 \mathrm{~km}$ do entroncamento, epífila de arbusto na mata, 19.III.2006, O. Yano \& M. A. R. Sousa 28801 (SP 382904); idem, epífila, próximo a rego d'água, em direção à Fazenda Portal do Sol e Lázaro, 19.III.2006, M. A. R. Sousa \& V. L. Gomes-Klein 106 (UFG 30184), 117 (UFG 30195).

Cresce associada com Aphanolejeunea truncatifolia Horik.

No Brasil, ocorre nos estados de Amapá, Amazonas, Bahia, Espírito Santo, Goiás (Alto Paraíso, Chapada dos Veadeiros), Mato Grosso, Mato Grosso do Sul, Minas Gerais, Pará, Paraíba, Paraná, Pernambuco, Rio de Janeiro, Rondônia, Roraima, Santa Catarina, São Paulo e Sergipe.

${ }^{*}$ Cololejeunea subcardiocarpa P. Tixier, Bradea 3(6): 39, 1980.

Ilustração: Tixier (1980).

Material examinado: Município de Pirenópolis, Parque Estadual da Serra dos Pireneus, na base do Morro do Cabeludo, epífila de arbusto na mata, 18.III.2006, O. Yano $\mathcal{E} M$. A. R. Sousa 28758 (SP 382862), 28761 p.p. (SP 382865), 28762 (SP 382866); idem, na estrada lateral de Pirenópolis a Cocalzinho, ca. $8 \mathrm{~km}$ a $9 \mathrm{~km}$ do entroncamento, 19.III.2006, O. Yano $\mathcal{E}$ M. A. R. Sousa 28786 (SP 382890).

Cresce associada com Aphanolejeunea truncatifolia Horik.

No Brasil, ocorre nos estados de Acre, Amazonas, Bahia, Ceará, Espírito Santo, Mato Grosso, Minas Gerais, Pará, Paraná, Pernambuco, Rio de Janeiro e São Paulo.

*Primeira referência para Goiás.

${ }^{*}$ Cololejeunea verwimpii P. Tixier, Cryptogamie, Bryol. Lichénol. 16(3): 230, 1995.

Ilustração: Tixier (1991).

Material examinado: Município de Pirenópolis, sítio Vereda de Cristal, perto da casa, epífila de arbusto perto do córrego no Chalé Beira Rio, 18.III.2006, O. Yano E M. A. R. Sousa 28595 (SP 379931); idem, Parque Estadual da Serra dos Pireneus, na base do Morro do Cabeludo, epífila de arbusto, 18.III.2006, O. Yano $\mathcal{E}$ M. A. R. Sousa 28720 (SP 382824); idem, epífila, na base da mata do Morro do Cabeludo, 18.III.2006, M. A. R. Sousa \& V. L. Gomes-Klein 56 (UFG 30134), 63 (UFG 30141), 64 (UFG 30142).

No Brasil, ocorre nos estados de Amazonas, Espírito Santo, Mato Grosso do Sul, Minas Gerais, Paraná, Rio Grande do Sul, Santa Catarina e São Paulo.

*Primeira referência para Goiás.

*Frullanoides densifolia Raddi, Critt. Brasil.: $14,1822$.

Ilustrações: Gradstein (1994) e LemosMichel (2001).

Material examinado: Município de Pirenópolis, Córrego da Barriguda, 1.040 m alt., sobre tronco de arbusto, 18.III.2006, O. Yano $\mathcal{E}$ M. A. R. Sousa 28605 (SP 379941); idem, na estrada para o Morro do Cabeludo, sobre tronco de arbusto, 18.III.2006, O. Yano \& M. A. R. Sousa 28637 (SP 379973); idem, Parque Estadual da Serra dos Pireneus, Morro do Cabeludo, sobre tronco de Clusiaceae, 18.III.2006, O. Yano \& $M$. A. R. Sousa 28667 (SP 380002); idem, epífila, na base da mata do Morro do Cabeludo, sobre tronco de Clusia, 18.III.2006, M. A. R. Sousa \& V. L. Gomes-Klein 60 (UFG 30138). 
No Brasil, ocorre nos estados de Amazonas, Bahia, Ceará, Espírito Santo, Minas Gerais, Pará, Paraná, Rio Grande do Sul, Rio de Janeiro, Santa Catarina e São Paulo.

*Primeira referência para Goiás.

Lejeunea flava (Sw.) Nees, Naturgesch. Eur. Leberm. 3: 277, 1838. Jungermannia flava Sw.

Ilustrações: Lemos-Michel (2001) e Reiner-Drehwald (2000).

Material examinado: Município de Pirenópolis, sítio Vereda de Cristal, perto da casa, sobre tronco perto do córrego no Chalé Beira Rio, 18.III.2006, O. Yano E M.A. R. Sousa 28596 (SP 379932); idem, Parque Estadual da Serra dos Pireneus, na base do Morro do Cabeludo, sobre pedra úmida, 18.III.2006, O. Yano $\mathcal{E} M$. A. R. Sousa 28739 (SP 382843); idem, na estrada lateral de Pirenópolis a Cocalzinho, ca. 8 $\mathrm{km}$ a $9 \mathrm{~km}$ do entroncamento, sobre tronco de arbusto na encosta, 19.III.2006, O. Yano $\mathcal{E} M$. A. R. Sousa 28765 (SP 382869); idem, na base do tronco de arbusto na encosta, 19.III.2006, O. Yano E M. A. R. Sousa 28766 (SP 382870); idem, na base do tronco de arbusto na mata, 19.III.2006, O. Yano E M. A. R. Sousa 28781 (SP 383885); idem, na base do tronco fino na mata, 19.III.2006, O. Yano E M. A. R. Sousa 28792 (SP 382896); idem, sobre tronco vivo, próximo a rego d'água que corta a estrada rumo à Fazenda Portal do Sol e Lázaro, 19.III.2006, M. A. R. Sousa \& V. L. Gomes-Klein 113 (UFG 30191).

No Brasil, ocorre no Distrito Federal e nos estados de Acre, Amazonas, Bahia, Ceará, Espírito Santo, Goiás (Alto Paraíso, Chapada dos Veadeiros), Mato Grosso, Mato Grosso do Sul, Minas Gerais, Pará, Paraíba, Paraná, Pernambuco, Rio Grande do Sul, Rio de Janeiro, Roraima, Santa Catarina, São Paulo e Sergipe.

*Lejeunea laetevirens Nees \& Mont. in Ramón de la Sagra, Hist. Phys. Bot. Plant. Cell. Cuba 9: 469, 1842.

Ilustração: Reiner-Drehwald (2000).

Material examinado: Município de Pirenópolis, Córrego da Barriguda, $1.040 \mathrm{~m}$ alt., sobre tronco de arbusto na margem do córrego, 18.III.2006, O. Yano \& M. A. R. Sousa 28600 (SP 379936); idem, na estrada para o Morro do Cabeludo, sobre tronco de arbusto, 18.III.2006, O. Yano \& M. A. R. Sousa
28628 (SP 379964); idem, Parque Estadual da Serra dos Pireneus, na base do Morro do Cabeludo, 18.III.2006, O. Yano \& M. A. R. Sousa 28692 p.p. (SP 380027); idem, sobre tronco de arbusto, 18.III.2006, O. Yano E M. A. R. Sousa 28712 (SP 380047); idem, na estrada lateral de Pirenópolis a Cocalzinho, ca. $8 \mathrm{~km}$ a $9 \mathrm{~km}$ do entroncamento, na base do tronco de arbusto na encosta, 19.III.2006, O. Yano \& M. A. R. Sousa 28769 (SP 382873), 28772 (SP 382876); idem, corticícola, próximo a riacho, na estrada para o Sítio Vereda de Cristal, 18.III.2006, M. A. R. Sousa \& V. L. Gomes-Klein 48 (UFG 30126); idem, corticícola, na base da mata do Morro do Cabeludo, 18.III.2006, M. A. R. Sousa \& V. L. Gomes-Klein 87 p.p. (UFG 30165); idem, sobre tronco, margem do rego d'água, em direção à Fazenda Portal do Sol e Lázaro, 19.III.2006, M. A. R. Sousa \& V. L. Gomes-Klein 99 (UFG 30177).

Cresce associada com Cheilolejeunea clausa (Nees \& Mont.) Schust. e Schiffneriolejeunea polycarpa (Nees) Gradst.

No Brasil, ocorre no Distrito Federal, na Ilha Fernando de Noronha e nos estados de Acre, Alagoas, Amapá, Amazonas, Bahia, Ceará, Espírito Santo, Mato Grosso, Mato Grosso do Sul, Minas Gerais, Pará, Paraíba, Pernambuco, Rio de Janeiro, Roraima, Santa Catarina, São Paulo e Sergipe.

*Primeira referência para Goiás.

*Lejeunea phyllobola Nees \& Mont. in Ramón de la Sagra, Hist. Fis. Pol. Natur. Bot. Cuba 9: 471, 1842.

Ilustração: Reiner-Drehwald (2000).

Material examinado: Município de Pirenópolis, Parque Estadual da Serra dos Pireneus, na base do Morro do Cabeludo, sobre tronco de arbusto, 18.III.2006, O. Yano $\mathcal{E}$ M. A. R. Sousa 28723 (SP 382827).

No Brasil, ocorre no Distrito Federal e nos estados de Acre, Alagoas, Amazonas, Bahia, Ceará, Espírito Santo, Mato Grosso, Mato Grosso do Sul, Pará, Rio Grande do Sul, Rio de Janeiro, Santa Catarina e São Paulo.

*Primeira referência para Goiás.

Lopholejeunea nigricans (Lindenb.) Schiffn., Consp. Hepat. Archip. Ind.: 293, 1898. Lejeunea nigricans Lindenb. in Gottsche et al.

Ilustração: Gradstein (1994). 
Material examinado: Município de Pirenópolis, na estrada para o Morro do Cabeludo, sobre pedra no riacho, 18.III,2006, O. Yano $\mathcal{E}$ M. A. R. Sousa 28625 (SP 379961).

No Brasil, ocorre no Distrito Federal e nos estados de Acre, Amazonas, Bahia, Ceará, Espírito Santo, Goiás (Chapada dos Veadeiros), Mato Grosso, Mato Grosso do Sul, Minas Gerais, Pará, Paraná, Pernambuco, Rio de Janeiro, Santa Catarina e São Paulo.

*Lopholejeunea subfusca (Nees) Schiffn., Bot. Jahrb. Syst. 23: 593, 1897. Jungermannia subfusca Nees.

Ilustração: Schuster (1980).

Material examinado: Município de Pirenópolis, Córrego da Barriguda, sobre pedra exposta, 18.III.2006, O. Yano \& M. A. R. Sousa 28643 (SP 379979); idem, na estrada lateral de Pirenópolis a Cocalzinho, ca. $8 \mathrm{~km}$ a $9 \mathrm{~km}$ do entroncamento, na base de tronco de arbusto na encosta, 19.III.2006, O. Yano \& M. A. R. Sousa 28774 (SP 382878); idem, sobre tronco de Melastomataceae na mata, 19.III.2006, O. Yano E M. A. R. Sousa 28777 (SP 382881).

No Brasil, ocorre no Distrito Federal e nos estados de Acre, Amazonas, Amapá, Bahia, Ceará, Espírito Santo, Mato Grosso, Mato Grosso do Sul, Minas Gerais, Pará, Paraíba, Pernambuco, Rio de Janeiro, Rondônia, Roraima, Santa Catarina e São Paulo.

*Primeira referência para Goiás.

*Mastigolejeunea plicatiflora (Spruce) Steph., Spec. Hepat. 4: 766, 1912. Lejeunea plicatiflora Spruce.

Ilustração: Bastos (2004).

Material examinado: Município de Pirenópolis, na estrada para o Morro do Cabeludo, sobre tronco de arbusto na trilha, 18.III.2006, O. Yano $\mathcal{E}$ M. A. R. Sousa 28636 (SP 379972); idem, Parque Estadual da Serra dos Pireneus, na base do Morro do Cabeludo, nos galhos de arbusto, 18.III.2006, O. Yano E M. A. R. Sousa 28689 (SP 380024).

No Brasil, ocorre nos estados de Acre, Amazonas, Bahia, Espírito Santo, Pará, Roraima e São Paulo.

*Primeira referência para Goiás.

${ }^{*}$ Microlejeunea bullata (Tayl.) Steph., Hedwigia 29: 90, 1890. Lejeunea bullata Tayl.
Ilustração: Schuster (1980, como Microlejeunea ulicina var. bullata Tayl.).

Material examinado: Município de Pirenópolis, Córrego da Barriguda, a 1.040 m alt., sobre pedra perto do córrego no Chalé Beira Rio, 18.III.2006, O. Yano E M. A. R. Sousa 28601 p.p. (SP 379937).

Cresce associada com Fabronia ciliaris var. polycarpa (Hook.) Buck.

No Brasil, ocorre no Distrito Federal e nos estados de Acre, Amazonas, Bahia, Ceará, Espírito Santo, Mato Grosso, Mato Grosso do Sul, Minas Gerais, Paraná, Pernambuco, Rio de Janeiro, Roraima, São Paulo e Sergipe.

*Primeira referência para Goiás.

* Microlejeunea epiphylla Bischler in Bischler et al., Nova Hedwigia 5(1-2): 378, 1967.

Ilustração: Gradstein \& Costa (2003).

Material examinado: Município de Pirenópolis, Parque Estadual da Serra dos Pireneus, epífita, próximo a rego d'água que corta a estrada rumo à Cachoeira de São Lázaro, 19.III.2006, M. A. R. Sousa \& V. L. Gomes-Klein 118 p.p. (UFG 30196).

Cresce associada com Schiffneriolejeunea polycarpa (Nees) Gradst.

No Brasil, ocorre nos estados de Amapá, Bahia, Ceará, Espírito Santo, Mato Grosso do Sul, Minas Gerais, Pará, Paraíba, Pernambuco, São Paulo e Sergipe.

*Primeira referência para Goiás.

Schiffneriolejeunea polycarpa (Nees) Gradst., J. Hattori Bot. Lab. 38: 355, 1985. Jungermannia polycarpa Nees in Martius.

Ilustração: Gradstein (1994).

Material examinado: Município de Pirenópolis, Parque Estadual da Serra dos Pireneus, margem do Córrego da Barriguda, epífita, 2.X.2005, M. A. R. Sousa \& A. Moris 22 p.p. (UFG 30098); idem, na estrada para o Morro do Cabeludo, sobre tronco de arbusto, 18.III.2006, O. Yano $\mathcal{E}$ M. A. R. Sousa 28633 (SP 379969); idem, Parque Estadual da Serra dos Pireneus, Morro do Cabeludo, sobre tronco de arbusto, 18.III.2006, O. Yano E M. A. R. Sousa 28683 (SP 380018), 28647 p.p. (SP 379983); idem, na base do Morro do Cabeludo, sobre tronco de árvore, 18.III.2006, O. Yano E M. A. R.Sousa 28691 p.p. (SP 380026); idem, sobre galhos finos, 18.III.2006, O. Yano $\mathcal{E}$ 
M. A. R. Sousa 28703 (SP 380036); idem, sobre tronco podre, 18.III.2006, O. Yano $\mathcal{E} M$. A. R. Sousa 28704 (SP 380039); idem, sobre tronco de arbusto, 18.III.2006, O. Yano \& M. A. R. Sousa 28711 (SP 380046), 28715 (SP 382819), 28731 (SP 382835); idem, sobre galhos finos de arbusto, 18.III.2006, O. Yano \& M. A. R. Sousa, 28719 (SP $382823), 28735$ p.p. (SP 382839); idem, sobre tronco de arbusto na mata, 18.III.2006, O. Yano $\mathcal{E}$ M. A. R. Sousa 28722 (SP 382826), 28745 (SP 382849), 28749 (SP 382853), 28751 (SP 382855), 28753 (SP 382857); idem, nos galhos de arbusto, 18.III.2006, O. Yano \& M. A. R. Sousa 28725 (SP 382829); idem, na estrada lateral de Pirenópolis a Cocalzinho, ca. $8 \mathrm{~km}$ a $9 \mathrm{~km}$ do entroncamento, na base do tronco de arbusto na mata, 19.III.2006, O. Yano \& M. A. R. Sousa 28782 (SP 382886), 28796 (SP 382899), 28804 (SP 382907); idem, rupícola, na base da mata do Morro do Cabeludo, 18.III.2006, M. A. R. Sousa \& V. L. Gomes-Klein 79 (UFG 30157), 81 p.p. (UFG 30159), 83 (UFG 30161), 87 p.p. (UFG 30165), 89 (UFG 30167); idem, epífita, próximo a rego d'água que corta a estrada rumo à Cachoeira de São Lázaro, 19.III.2006, M. A. R. Sousa 118 p.p. (UFG 30169).

Cresce associada com Cheilolejeunea rigidula (Nees \& Mont.) Schust., Fissidens prionodes Mont., Lejeunea laetevirens Nees \& Mont., Metzgeria conjugata Lindb., M. lechleri Steph., Microlejeunea epiphylla Bischler, Plagiochila corrugata (Nees) Nees \& Mont. e Sematophyllum subsimplex (Hedw.) Mitt.

No Brasil, ocorre no Distrito Federal e nos estados de Acre, Amazonas, Bahia, Ceará, Espírito Santo, Goiás (Chapada dos Veadeiros), Mato Grosso, Mato Grosso do Sul, Minas Gerais, Pará, Paraíba, Pernambuco, Rio Grande do Sul, Rio de Janeiro, Roraima, Santa Catarina, São Paulo e Sergipe.

*Taxilejeunea pterigonia (Lehm. \& Lindenb.) Schiffn. in Engler \& Prantl, Natürl. Pflanzenfam. 1(3): 125, 1893. Jungermannia pterigonia Lehm. \& Lindenb. in Lehmann.

Ilustração: Reiner-Drehwald (2000).

Material examinado: Município de Pirenópolis, Parque Estadual da Serra dos Pireneus, na base do Morro do Cabeludo, sobre tronco de arbusto na mata, 18.III.2006, O. Yano E M. A. R. Sousa 28748 (SP 382862); idem, na base da mata do Morro do Cabeludo, em rocha dentro do riacho, 18.III.2006, M. A. R. Sousa \& V. L. Gomes-Klein 57 p.p. (UFG 30135); idem, rupícola, na base da mata do Morro do Cabeludo, 18.III.2006, M. A. R. Sousa \& V. L. Gomes-Klein 85 p.p. (UFG 30163).

Cresce associada com Cheilolejeunea rigidula (Nees \& Mont.) Schust., Lophocolea platensis C. Massal. e Thuidium delicatulum (Hedw.) B.S.G.

No Brasil, ocorre nos estados de Amazonas, Bahia, Espírito Santo, Minas Gerais, Rio Grande do Sul, Rio de Janeiro, Santa Catarina e São Paulo.

*Primeira referência para Goiás.

LePIDOZIACEAE

*Bazzania pallide-virens (Steph.) Fulf., Annales Crypt. Phytopath. 3: 42, 1946. Mastigobryum pallide-virens Steph.

Ilustrações: Fulford (1963) e Oliveira e Silva \& Yano (2000).

Material examinado: Município de Pirenópolis, Parque Estadual da Serra dos Pireneus, na base do Morro do Cabeludo, sobre rocha úmida, 18.III.2006, O. Yano \& $M$. A. R. Sousa 28717 (SP 382821).

No Brasil, ocorre nos estados de Amazonas, Ceará, Mato Grosso, Rio de Janeiro e Roraima.

*Primeira referência para Goiás.

*Bazzania stolonifera (Sw.) Trevis., Mem. C. Ist. Lombardo Cl. Sci. ser. 3, 4: 415, 1877. Jungermannia stolonifera Sw.

Ilustrações: Fulford (1963) e Oliveira e Silva \& Yano (2000).

Material examinado: Município de Pirenópolis, Parque Estadual da Serra dos Pireneus, na base do Morro do Cabeludo, sobre rocha úmida, 18.III.2006, O. Yano \& M. A. R. Sousa 28726 p.p. (SP 382830).

Cresce associada com Plagiochila simplex (Sw.) Lindenb.

No Brasil, ocorre nos estados de Bahia, Espírito Santo, Minas Gerais, Rio de Janeiro e São Paulo.

*Primeira referência para Goiás.

Kurzia brasiliensis (Steph.) Grolle, Revue Bryol. Lichénol. 32: 175, 1963. Psiloclada brasiliensis Steph. 
Ilustração: Fulford (1966, como Lepidozia brasiliensis Steph.).

Material examinado: Município de Pirenópolis, Parque Estadual da Serra dos Pireneus, na base do Morro do Cabeludo, sobre pedra úmida, 18.III.2006, O. Yano $\mathcal{E} M$. A. R. Sousa 28690 (SP 380025).

No Brasil, ocorre no Distrito Federal e nos estados de Bahia, Espírito Santo, Goiás (Chapada dos Veadeiros), Minas Gerais, Rio Grande do Sul, Rio de Janeiro, Roraima, Santa Catarina e São Paulo.

Kurzia capillaris (Sw.) Grolle, Revue Bryol. Lichénol. 32: 178, 1963. Jungermannia capillaris Sw.

Ilustração: Gradstein \& Costa (2003).

Material examinado: Município de Pirenópolis, Parque Estadual da Serra dos Pireneus, Morro do Cabeludo, sobre pedra úmida, 18.III.2006, O. Yano \& M. A. R. Sousa 28673 p.p. (SP 380008); idem, na base do Morro do Cabeludo, sobre pedra úmida, 18.III.2006, O. Yano E M. A. R. Sousa 28705 p.p. (SP 380040); idem, no solo úmido, 18.III.2006, O. Yano E M. A. R. Sousa 28728 p.p. (SP 382832).

Cresce associada com Campylopus reflexisetus Herzog, Odontoschisma brasiliensis Steph., Symphyogyna brasiliensis (Nees) Nees \& Mont., Syrrhopodon prolifer Schwägr. e Zoopsidela integrifolia (Spruce) Schust.

No Brasil, ocorre no Distrito Federal e nos estados de Amazonas, Bahia, Ceará, Goiás (Alto Paraíso, Chapada dos Veadeiros), Mato Grosso, Minas Gerais, Rio de Janeiro e São Paulo.

Paracromastigum pachyrhizon (Nees in Martius) Fulf., Mem. New York Bot. Gard. 11(3): 390, 1968. Jungermannia pachyrhiza Nees in Martius.

Ilustrações: Fulford (1968) e Gradstein \& Costa (2003).

Material examinado: Município de Pirenópolis, na estrada lateral de Pirenópolis a Cocalzinho, ca. $8 \mathrm{~km}$ a $9 \mathrm{~km}$ do entroncamento, no barranco perto da estrada, 19.III.2006, O. Yano E M. A. R. Sousa 28805 p.p. (SP 382908); idem, no solo úmido da mata perto da estrada, 19.III.2006, O. Yano E M.A.R Sousa 28814 (SP 382917); idem, no barranco úmido da mata, 19.III.2006, O. Yano E M. A. R. Sousa 28815 (SP 382918).
Cresce associada com Jungermannia hyalina Lyell.

No Brasil, ocorre nos estados de Espírito Santo, Goiás (Alto Paraíso, Chapada dos Veadeiros, Rio Preto), Minas Gerais, Santa Catarina e São Paulo.

Telaranea nematodes (Gott. ex Aust.) Howe, Bull. Torrey. Bot. Club 6: 302, 1879. Cephalozia nematodes Gott. ex Aust.

Ilustrações: Fulford (1966, como Telaranea sejuncta (Ångstr.) S. Arnell) e Schuster \& Blomquist (1955).

Material examinado: Município de Pirenópolis, Parque Estadual da Serra dos Pireneus, na base do Morro do Cabeludo, em solo úmido, 18.III.2006, O. Yano \& M. A. R. Sousa 28729 p.p. (SP 382833).

Cresce associada com Cephalozia crassifolia (Lindenb. \& Gott.) Fulf.

No Brasil, ocorre no Distrito Federal e nos estados de Acre, Amazonas, Bahia, Ceará, Espírito Santo, Goiás (Mineiros), Mato Grosso, Mato Grosso do Sul, Minas Gerais, Rio Grande do Sul, Rio de Janeiro, Roraima, Santa Catarina, São Paulo e Sergipe.

*Zoopsidella integrifolia (Spruce) Schust., Bull. Natl. Sci. Mus. Tokyo, n.s., 12: 666, 1969. Cephalozia integrifolia Spruce.

Ilustrações: Fulford (1968, como Zoopsis integrifolia (Spruce) Steph.) e Gradstein \& Costa (2003).

Material examinado: Município de Pirenópolis, Parque Estadual da Serra dos Pireneus, Morro do Cabeludo, sobre pedra úmida, 18.III.2006, O. Yano \& M. A. R. Sousa 28673 p.p. (SP 380008).

Cresce associada com Campylopus reflexisetus Herzog, Kurzia capillaris (Sw.) Grolle e Symphyogyna brasiliensis (Nees) Nees \& Mont.

No Brasil, ocorre no Distrito Federal e nos estados de Amazonas, Bahia, Mato Grosso, Minas Gerais, Pará, São Paulo e Sergipe.

*Primeira referência para Goiás.

\section{Metzgeriaceae}

*Metzgeria conjugata Lindb., Acta Soc. Sci. Fenn. 10: 495, 1875. 
ta (1999).

Ilustrações: Costa \& Moura (1996) e Cos-

Material examinado: Município de Pirenópolis, Parque Estadual da Serra dos Pireneus, Morro do Cabeludo, sobre tronco de arbusto, 18.III.2006, O. Yano E M. A. R. Sousa 28647 p.p. (SP 379983).

Cresce associada com Schiffneriolejeunea polycarpa (Nees) Gradst.

No Brasil, ocorre nos estados de Ceará, Espírito Santo, Minas Gerais, Paraná, Pernambuco, Rio Grande do Sul, Rio de Janeiro e São Paulo.

*Primeira referência para Goiás.

${ }^{*}$ Metzgeria convoluta Steph. Spec. Hepat. 1: $288,1899$.

Ilustração: Costa (1999).

Material examinado: Município de Pirenópolis, Parque Estadual da Serra dos Pireneus, sobre tronco vivo, próximo a rego d'água, em direção à Fazenda Portal do Sol e Lázaro, 19.III.2006, M. A. R. Sousa \& V. L. Gomes-Klein 104 (UFG 30182).

Ocorre apenas no Brasil, nos estados de Bahia, Paraná, Pernambuco, Rio Grande do Sul, Rio de Janeiro, Santa Catarina e São Paulo.

*Primeira referência para Goiás.

Metzgeria lechleri Steph., Spec. Hepat. 1: 290, 1899.

Ilustrações: Costa (1999), Gradstein \& Costa (2003) e Kuwahara (1986).

Material examinado: Município de Pirenópolis, Parque Estadual da Serra dos Pireneus, margem do Córrego da Barriguda, epífita, 2.X.2005, M. A. R. Sousa E A. Moris 22 p.p. (UFG 30098); idem, na estrada lateral de Pirenópolis a Cocalzinho, ca. $8 \mathrm{~km}$ a $9 \mathrm{~km}$ do entroncamento, sobre tronco de arbusto perto do riacho, 19.III.2006, O. Yano \& M. A. R. Sousa 28800 (SP 382903); idem, sobre tronco de arbusto na mata, 19.III.2006, O. Yano \& M. A. R. Sousa 28803 p.p. (SP 382906); idem, sobre tronco vivo, na estrada em direção à Cachoeira de São Lázaro, 19.III.2006, M. A. R. Sousa \& V. L. Gomes-Klein 116 (UFG 30194).

Cresce associada com Cheilolejeunea rigidula (Mont.) R.M. Schust., Fissidens prionodes Mont., Schiffnerioljeuenea polycarpa (Nees) Gradst., Sematophyllum subpinnatum (Brid.) Britt. e S. subsimplex (Hedw.) Mitt.
No Brasil, ocorre no Distrito Federal e nos estados de Ceará, Espírito Santo, Goiás (Jataí; Parque Nacional da Chapada dos Veadeiros; Serra do Silêncio; Sete Lagoas, Rio Preto), Minas Gerais, Paraná, Pernambuco, Rio Grande do Sul, Rio de Janeiro, Santa Catarina e São Paulo.

\section{Palaviciniaceae}

*Symphyogyna aspera Steph. in Mc Cormick, Bot. Gaz. 58: 403, 1914.

Ilustrações: Hell (1969) e Oliveira e Silva \& Yano (2000).

Material examinado: Município de Pirenópolis, Parque Estadual da Serra dos Pireneus, quase dentro do Córrego da Barriguda, rupícola, 2.X.2005, M. A. R. Sousa E A. Moris 10 (UFG 30087); idem, na base do Morro do Cabeludo, em solo úmido, 18.III.2006, O. Yano E M. A. R. Sousa 28724 (SP 382828); idem, rupícola, dentro do rio que corta a base da mata do Morro do Cabeludo, 18.III.2006, M. A. R. Sousa \& V. L. Gomes-Klein 76 p.p. (UFG 30154), 77 p.p. (UFG 30155), 82 (UFG 30160).

Cresce associada com Lophocolea platensis C. Massal.

No Brasil, ocorre no Distrito Federal e nos estados de Amazonas, Bahia, Ceará, Espírito Santo, Mato Grosso, Mato Grosso do Sul, Minas Gerais, Pará, Pernambuco, Rio Grande do Sul, Rio de Janeiro, Santa Catarina e São Paulo.

*Primeira referência para Goiás.

Symphyogyna brasiliensis (Nees) Nees \& Mont., Ann. Sci. Nat. Bot. ser. 2, 5: 67, 1836. Jungermannia brasiliensis Nees.

Ilustrações: Gradstein \& Costa (2003) e Hell (1969).

Material examinado: Município de Pirenópolis, Parque Estadual da Serra dos Pireneus, quase dentro do Córrego da Barriguda, rupícola, 2.X.2005, M. A. R. Sousa E A. Moris 14 (UFG 30092); idem, Morro do Cabeludo, sobre pedra úmida, 18.III.2006, O. Yano $\mathcal{E} M$. A. R. Sousa 28673 p.p. (SP 380008).

Cresce associada com Campylopus reflexisetus Herzog, Kurzia capillaris (Sw.) Grolle e Zoopsidella integrifolia (Spruce) Schust.

No Brasil, ocorre no Distrito Federal e nos estados de Bahia, Ceará, Espírito Santo, 
Goiás (Chapada dos Veadeiros, Serra Dourada), Mato Grosso, Minas Gerais, Paraná, Rio Grande do Sul, Rio de Janeiro, Rondônia, Roraima, Santa Catarina e São Paulo.

*Symphyogyna podophylla (Thunb.) Mont. \& Nees in Gottsche et al., Syn. Hepat.: 481, 1846. Jungermannia podophylla Thunb. Hell (1969).

Ilustrações: Gradstein \& Costa (2003) e

Material examinado: Município de Pirenópolis, Parque Estadual da Serra dos Pireneus, na base do Morro do Cabeludo, no solo úmido da mata, 18.III.2006, O. Yano $\mathcal{E}$ M. A. R. Sousa 28734 (SP 382838).

No Brasil, ocorre nos estados de Amazonas, Espírito Santo, Minas Gerais, Rio de Janeiro, Santa Catarina e São Paulo.

*Primeira referência para Goiás.

\section{Plagiochilaceae}

*Plagiochila corrugata (Nees) Nees \& Mont., Ann. Sci. Nat. Bot. ser. 2, 5: 52, 1836. Jungermannia corrugata Nees in Martius.

Ilustrações: Lemos-Michel (2001) e Lindenberg (1839-1844).

Material examinado: Município de Pirenópolis, na estrada para o Morro do Cabeludo, na base do tronco de arbusto, 18.III.2006, O. Yano E M. A. R. Sousa 28630 (SP 379966); idem, epífita, na base da mata do Morro do Cabeludo, 18.III.2006, M. A. R. Sousa \& V. L. Gomes-Klein 81 p.p. (UFG 30159), 102 (UFG 30180).

Cresce associada com Schiffneriolejeunea polycarpa (Nees) Gradst.

No Brasil, ocorre no Distrito Federal e nos estados de Acre, Bahia, Espírito Santo, Minas Gerais, Paraná, Pernambuco, Rio Grande do Sul, Rio de Janeiro, Santa Catarina e São Paulo.

*Primeira referência para Goiás.

*Plagiochila disticha (Lehm. \& Lindenb.) Lindenb., Spec. Hepat. fasc. 4: 108, 1840. Jungermannia disticha Lehm. \& Lindenb. in Lehmann.

Ilustrações: Heinrichs \& Gradstein (2000), Lemos-Michel (2001) e Lindenberg (1839-1844).

Material examinado: Município de Pirenópolis, Parque Estadual da Serra dos Pireneus, na base do Morro do Cabeludo, sobre tronco de arbusto, 18.III.2006, O. Yano $\mathcal{E}$ M. A. R. Sousa 28693 (SP 380028).
No Brasil, ocorre no Distrito Federal e nos estados de Acre, Alagoas, Amapá, Amazonas, Bahia, Ceará, Espírito Santo, Mato Grosso, Mato Grosso do Sul, Minas Gerais, Pará, Paraíba, Pernambuco, Rio Grande do Sul, Rio de Janeiro, Roraima, Santa Catarina e São Paulo.

*Primeira referência para Goiás.

*Plagiochila macrostachya Lindenb., Spec. Hepat. fasc. 2-4: 75, 1840.

Ilustrações: Gradstein \& Costa (2003) e Lindenberg (1839-1844).

Material examinado: Município de Pirenópolis, Parque Estadual daSerra dos Pireneus, Morro do Cabeludo, sobre pedra úmida, 18.III.2006, O. Yano E M. A. R. Sousa 28707 (SP 380042); idem, rupícola, na base da mata do Morro do Cabeludo, 18.III.2006, M. A. R. Sousa \& V. L. GomesKlein 65 (UFG 30143), 78 (UFG 30156).

No Brasil, ocorre nos estados de Espírito Santo, Minas Gerais, Rio de Janeiro, Santa Catarina e São Paulo.

*Primeira referência para Goiás.

*Plagiochila martiana (Nees) Lindenb., Spec. Hepat. fasc. 1: 12, 1839. Jungermannia martiana Nees.

Ilustrações: Lemos-Michel (2001), Lindenberg (1839-1844) e Oliveira e Silva \& Yano (2000).

Material examinado: Município de Pirenópolis, Córrego da Barriguda, sobre tronco de arbusto, 18.III.2006, O. Yano \& M. A. R. Sousa 28641 (SP 379977); idem, Parque Estadual da Serra dos Pireneus, na base do Morro do Cabeludo, sobre tronco de arbusto, 18.III.2006, O. Yano E M. A. R. Sousa 28733 (SP 382837).

No Brasil, ocorre no Distrito Federal e nos estados de Acre, Alagoas, Bahia, Ceará, Espírito Santo, Mato Grosso, Mato Grosso do Sul, Minas Gerais, Pará, Paraná, Pernambuco, Rio Grande do Sul, Rio de Janeiro, Santa Catarina e São Paulo.

*Primeira referência para Goiás.

Plagiochila simplex (Sw.) Lindenb., Spec. Hepat. fasc. 2-4: 54, 1840. Jungermannia simplex Sw.

Ilustrações: Gradstein \& Costa (2003), Heinrichs et al. (1998) e Lindenberg (1839-1844).

Material examinado: Município de Pirenópolis, Parque Estadual da Serra dos Pi- 
reneus, na base do Morro do Cabeludo, sobre pedra úmida, 18.III.2006, O. Yano \& M. A. R. Sousa 28726 p.p. (SP 382830).

Cresce associada com Bazzania stolonifera (Sw.) Trevis.

No Brasil, ocorre nos estados de Amazonas, Bahia, Goiás (Alto Paraíso, Chapada dos Veadeiros), Minas Gerais, Pará, Pernambuco, Rio de Janeiro e São Paulo.

\section{RADULACEAE}

*Radula quadrata Gott. in Gottsche et al., Syn. Hepat.: 255, 1844.

Ilustrações: Castle (1964-1965) e Gradstein $\&$ Costa (2003).

Material examinado: Município de Pirenópolis, na estrada lateral de Pirenópolis a Cocalzinho, ca. $8 \mathrm{~km}$ a $9 \mathrm{~km}$ do entroncamento, na base do tronco de arbusto na encosta, 19.III.2006, O. Yano E M. A. R. Sousa 28770 (SP 382874), 28773 (SP 382877).

No Brasil, ocorre nos estados de Bahia, Minas Gerais, Pará, Paraná, Rio Grande do Sul, Rio de Janeiro e São Paulo.

*Primeira referência para Goiás.

\section{Agradecimentos}

Ao Conselho Nacional de Desenvolvimento Científico e Tecnológico (CNPq) pelo apoio nos custos de transporte e hospedagem durante as viagens de coleta para a execução do Projeto "Estudo florístico da Serra dos Pireneus, Goiás, Brasil" (Processo N. 647336/572162515) e pela bolsa concedida através do Programa Institucional de Bolsas de Iniciação Científica - PIBIC.

\section{ReferÊnCIAS}

Bastos, C. J. P. 2004. Lejeuneaceae (Marchantiophyta) no estado da Bahia, Brasil. São Paulo, Instituto de Biociências, Universidade de São Paulo, 442 p, Tese de Doutorado.

Brotherus, V. F. von. 1895. Beiträge zur Kenntniss der brasilianischen Moosflora. Hedwigia 34: 117-131.

Castle, H. 1964-1965. A revision of the genus Radula. Part II. sub genus Acroradula. Section 9. Densifoliae. Revue Bryol. Lichénol. 33: 328-397.
Costa, D. P. 1999. Metzgeriaceae (Metzgeriales, Hepatophyta) no Brasil. São Paulo, Universidade de São Paulo, 261 p, Tese de Doutorado.

Costa, D. P. \& A. C. Moura. 1996. Metzgeriaceae (Hepaticopsida) de Nova Friburgo, estado do Rio de Janeiro. Hoehnea 23: 103-122.

Crandall-Stotler, B. \& R. E. Stotler. 2000. Morphology and classification of the Marchantiophyta. In: A. J. Shaw \& B. Goffinet (Eds), Bryophytes biology. Cambridge, Cambridge University Press.

Fulford, M. H. 1963. Manual of the leafy Hepaticae of Latin America. Part I. Mem. New York Bot. Gard. 11: 1-172.

Fulford, M. H. 1966. Manual of the leafy Hepaticae of Latin America. Part II. Mem. New York Bot. Gard. 11: 173-276.

Fulford, M. H. 1968. Manual of the leafy Hepaticae of Latin America. Part III. Mem. New York Bot. Gard. 11: 277-392.

Fulford, M. H. 1976. Manual of the leafy Hepaticae of Latin America. Part IV. Mem. New York Bot. Gard. 11: 393-535.

Gradstein, S. R. 1994. Lejeuneaceae: Ptychanteae, Brachiolejeuneae. Flora Neotrop. Monogr. 62: 1-216.

Gradstein, S. R. \& D. P. Costa. 2003. The Hepaticae and Anthocerotae of Brazil. Mem. New York Bot. Gard. 87: 1-318.

Gradstein, S. R., A. Schäfer-Verwimp \& D. P. Costa. 2005. The liverworts (Marchantiophyta) of the state of Goiás, Brazil. Rev. Biol. Neotrop. 2: 75-108.

Hässel de Menéndez, G. G. 1962. Estudio de las Anthocerotales y Marchantiales de la Argentina. Opera Lilloana 7: 1-297.

Heinrichs, J. \& S. R. Gradstein. 2000. A revision of Plagiochila sect. Crispatae and sect. Hypnoides (Hepaticae) in the Neotropics. I. Plagiochila disticha, P. montagnei and P. raddiana. Nova Hedwigia 70: 161-184.

Heinrichs, J., S. R. Gradstein \& R. Grolle. 1998. A revision of the Neotropical species of Plagiochila (Dumort.) Dumort. (Hepaticae) described by Olof Swartz. J. Hattori Bot. Lab. 85: 1-32. 
Hell, K. G. 1969. Briófitas talosas dos arredores da cidade de São Paulo, Brasil. Bol. Univ. São Paulo, Bot. 25: 1-187.

Kuwahara, Y. 1986. The Metzgeriaceae of the Neotropics. Bryoph. Bibl. 28: 1-254.

Lemos-Michel, E. 2001. Hepáticas epifíticas sobre o pinheiro-brasileiro no Rio Grande do Sul. Editora da Universidade Federal do Rio Grande do Sul, Porto Alegre, 191 pp.

Lindenberg, J. B. G. 1839-1844. Monographia Hepaticarum generis Plagiochilae. Henry \& Cohen, Bonn.

Oliveira e Silva, M. I. M. N. \& O. Yano. 2000. Anthocerotophyta e Hepatophyta de Mangaratiba e Angra dos Reis, Rio de Janeiro, Brasil. Bol. Inst. de Bot. São Paulo 13: 1-102.

Reiner-Drehwald, M. E. 2000. Las Lejeuneaceae (Hepaticae) de Misiones, Argentina VI. Lejeunea y Taxilejeunea. Trop. Bryol. 19: 81-131.

Schuster, R. M. 1969. The Hepaticae and Anthocerotae of North America East of the hundredth meridian. Columbia University Press, New York, v. 2, 1062 pp.

Schuster, R. M. 1980. The Hepaticae and Anthocerotae of North America East of the hundredth meridian. Columbia University Press, New York, v. 4, 1334 pp.

Schuster, R. M. \& H. L. Blomquist. 1955. A comparative study of Telaranea nematodes. Amer. J. Bot. 42: 588-593.

Stotler, R. E. 1970. The genus Frullania subgenus Frullania in Latin America. Nova Hedwigia 18: 397-555.

Stotler, R. E. \& B. Crandall-Stotler. 2005. A revised classification of the Anthocerotophyta and a checklist of the hornworts of North America, North of Mexico. Bryol. 108: 16-26.
Tixier, P. 1980. Contribution a l'étude du genre Cololejeunea (Lejeuneaceae). IX. Espèces nouvelles du sous genre Pedinolejeunea (Ben.) Mizutani en région néotropicale. Bradea 3: 35-44.

Tixier, P. 1985. Contribution à la connaissance des Cololejeuneoideae. Bryoph. Bibl. 27: 1-439.

Tixier, P. 1991. Bryophyta exotica 9. Quelques Lejeuneaceae (Hépatiques) nouvelles pour l'Amérique du Sud. Candollea 46: 267-302.

Váňa, J. 1975. Studien über die Jungermanniaoideae (Hepaticae). 7. Jungermannia subg. Plectocolea: Europäische und nordamerikanische Arten. Folia Geobot. Phytotax. 10: 67-99.

Vanden Berghen, C. 1976. Frullaniaceae (Hepaticae) africanae. Bull. Jard. Bot. Belg. 46: 1-220.

Yano, O. 1984. Checklist of Brazilian liverworts and hornworts. J. Hattori Bot. Lab. 56: 841-548.

Yano, O. 1989. An additional checklist of Brazilian bryophytes. J. Hattori Bot. Lab. 66: 371-434.

Yano, O. 1995. A new additional annotated checklist of Brazilian bryophytes. J. Hattori Bot. Lab. 78: 137-182.

Yano, O. 2006. Novas adições ao catálogo de briófitas brasileiras. Bol. Inst. Bot. São Paulo 17: 1-142.

Yano, O. \& D. P. Costa. 2000. Criptógamos: Briófitas. In: I. F. P. Campos (Coord), Flora dos estados de Goiás e Tocantins. Goiânia, Editora da Universidade Federal de Goiás. Col. Rizzo 5: 1-33.

Yano, O. \& Z. R. Mello. 1999. Frullaniaceae dos manguezais do litoral sul de São Paulo, Brasil. Iheringia, Sér. Bot. 52: 65-87. 